\title{
Generating Synthetic Datasets for Mobile Wireless Networks with SUMO
}

\author{
Afonso Oliveira \\ Teresa Vazão \\ INESC-ID / Instituto Superior Técnico / Universidade de Lisboa \\ Lisboa, Portugal \\ afonso.oliveira@tecnico.ulisboa.pt
}

\begin{abstract}
With the softwarization of mobile wireless networks comes automated control and management of network infrastructure. Machine learning solutions come as critical enablers to achieve efficient network control and management. However, these machine learning solutions need data to train. In some applications, as is the resource allocation in the edge, large datasets, including User Equipment (UE) mobility between cells and traffic activity, are required. These may be difficult to obtain due to privacy concerns. This work presents a synthetic dataset generator that aims at supporting research activities in these areas. The introduced dataset generator uses traces from a known urban mobility simulator, Simulation of Urban MObility (SUMO). It matches them with empirical radio signal quality and diverse traffic models to obtain large datasets that can validate machine learning solutions. From the introduced generator, we created a dataset in an urban scenario in the city of Berlin with more than $6 \mathrm{~h}$ of duration, containing more than 40000 UEs served by 21 cells.
\end{abstract}

\section{CCS CONCEPTS}

- Networks $\rightarrow$ Mobile networks; Network simulations; Network performance modeling.

\section{KEYWORDS}

datasets, mobile wireless networks, 5G, resource allocation, SUMO

\section{ACM Reference Format:}

Afonso Oliveira and Teresa Vazão. 2021. Generating Synthetic Datasets for Mobile Wireless Networks with SUMO. In Proceedings of the 19th ACM International Symposium on Mobility Management (MobiWac '21), November 22-26, 2021, Aliante, Spain. ACM, New York, NY, USA, 10 pages. https: //doi.org/10.1145/3479241.3486704

\section{INTRODUCTION}

With the introduction of network softwarization, a lot of the management of wireless networks is automated [1]. With the introduction of $5 \mathrm{G}$ and its NFV/SDN-based architecture [2], the opportunities for infrastructure management automation increase. These

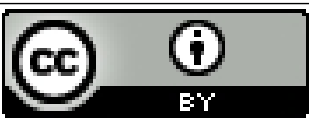

This work is licensed under a Creative Commons Attribution International 4.0 License.

MobiWac '21, November 22-26, 2021, Aliante, Spain.

(C) 2021 Copyright held by the owner/author(s).

ACM ISBN 978-1-4503-9079-8/21/11.

https://doi.org/10.1145/3479241.3486704 leave the door open for new solutions in infrastructure management, namely in the network's edge where resource allocation problems are one of the open issues being researched [3].

Machine learning has been successfully solving a vast array of problems from the health sector to engineering. These learning solutions can also be applied to solve some of the open issues in the network's edge. However, to validate these solutions, simple simulations are not enough since the learning models need to be trained with data that is not always available. For solutions that aim to provide resource allocation over an entire network with different base stations, a broad dataset with the alterations imposed by the different UEs in the network is required.

The ideal source of information for these problems would be mobile network operators, but since the data is ultimately from people's activity, privacy concerns arise. Data anonymization is possible but requires resources from the mobile operators and the possibility of complaints of insufficient anonymization makes the public publishing of these datasets an avoidable risk. For these reasons or others, some research works that managed to collect data from mobile network operators do not publish the data used [4] ${ }^{1}$ since they are bound by non-disclosure agreements.

So, there is an ongoing effort to generate data. Some work focus on continuous and long traces of individual datasets [5][6][4], while others focus on capturing a wider number of users often with data collection application installed in the terminals of a group of volunteers [7][8]. While being good for some applications, the first group does not provide a broad network knowledge with the occurrence of simultaneous handovers and connection quality changes of a wide set of UEs due to the low amount of simulation nodes. The second group gives a better answer to this problem by increasing the amount of UEs the data is collected from. However, the data from the different traces correspond to different time instances as it depends on when a volunteer uses the application for data collection. While one possibility would be to aggregate all traces as if they were being generated simultaneously, some traces may have some similarities. They may belong to the same volunteer making the same trip on different days.

In a wide array of research areas, synthetically generated datasets come as a possibility to replace data that is not easy to collect [9]. In the context of UE activity in the RAN of a mobile wireless network, only one synthetic dataset was found [8]. This dataset is generated using mathematical mobility models and constant bitrate traffic sources.

\footnotetext{
${ }^{1}$ In this specific citation a small dataset generated by the authors is published, however, they also state using a dataset from an operator they were not allowed publish.
} 
In this work, we present a synthetic dataset generator for wireless mobile networks that contains a realistic mobility generation and a diversified traffic generation model for the inclusion of different services in the foreseen $5 \mathrm{G}$ applications [10]. To provide a realistic mobility generation we use Simulation of Urban MObility (SUMO) [11], a renowned urban mobility simulator. Radio connection quality is simulated using empirical channel models [12][13]. A dataset using the city of Berlin as the basis is generated and made publicly available [14][15] along with the dataset generator itself [16].

The remaining of the paper is structured as follows. Section 2 provides related work on datasets for mobile wireless networks. Following that, Section 3 describes the introduced dataset generator. To give an example of a working dataset, Section 4 presents a generated dataset. Finally, Section 5 concludes the paper.

\section{RELATED WORK}

This section lists existing datasets in the area of wireless network communications. Then, we consider datasets of User Equipment (UE) connections to the cell sources, which is the focus of the dataset presented in this work.

The first group of datasets provides long samples of a small number of UEs controlled by the dataset authors. Bokani et al. [5] published a dataset of one UE in a vehicle making 71 trips of a $24 \mathrm{~km}$ course. The data contains Global Positioning System (GPS) and available bandwidth values with a time granularity of $10 \mathrm{~s}$. More recently, Raida et al. published traces of single UEs with complete radio information. One inside a building in an urban scenario [6], and three outdoor [4]: one in a rural environment and the other two in an urban environment. These works focus on long datasets of singular UEs in specific scenarios.

Other datasets have smaller traces per UE but try to include different scenarios and environments. This is the case of the dataset published by Hooft et al. where 40 traces of connections to an Long Term Evolution (LTE) infrastructure are registered. Each trace is 3 to 13 minutes long with data from GPS and achievable bandwidth with a 1 second granularity. The represented UEs did commutes in different vehicles, which adds to the dataset variety. A more detailed dataset is introduced by Meixner et al. [7] where 377 LTE traces are published with around 5 minutes per trace. Each entry has detailed information regarding the radio signal quality, position, and other measures with 100-millisecond granularity. Additionally, authors included in the application used for measuring a survey to assess the activity and environment associated with the user of each UE. Raca et al. [8] collected 135 LTE traces of around 15 minutes each. The dataset measurements are similar to those of Meixner with complete information but with the granularity of 1 second. The vehicle used by the user of each UE is also registered.

In their work Raca et al. also introduced a synthetic dataset [8] This synthetic dataset was generated in the $n s-3$ network simulator [17] using 100 UEs at a constant speed of $80 \mathrm{Km} / \mathrm{h}$ using a Gaussian Markov mobility model in an open space with seven cells. Traffic is composed of simple generators with a constant bitrate. To our knowledge, this is the only synthetically generated dataset introduced.

\section{DATASET GENERATOR FOR 3GPP SCENARIOS}

This section introduces the dataset generator introduced in this work. First, we present an overview of the generator. After that, we describe the individual modules that compose the generator.

\subsection{Dataset Generator overview}

Figure 1 illustrates the dataset generation process with the interaction between the different modules. The dataset generator goal is to generate three main types of traces:

- SNR traces - one trace per UE, representing the position and Signal-to-Noise Ratio (SNR) information at each instant of time.

- Traffic traces - one per UE, representing the amount of information it sends at each instant of time.

- Aggregated trace - an aggregation of SNR and traffic traces over time windows with a pre-specified size.

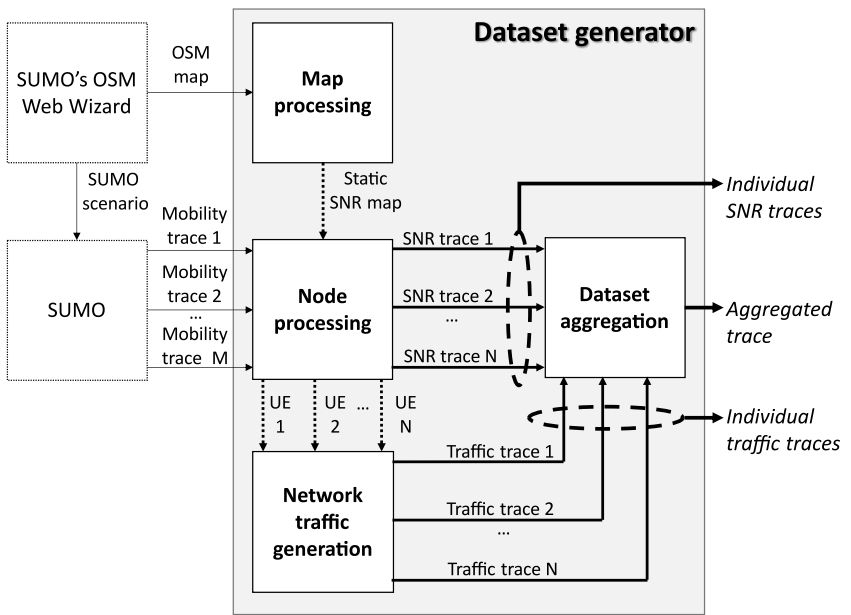

Figure 1: SNR and traffic synthetic trace generation

We used two tools of the Simulation of Urban Mobility (SUMO) framework: the simulator, SUMO [11], because it simulates the movement of pedestrians and vehicles with predetermined itineraries, and the Open Street Maps (OSM) Web Wizard. After all, it allows us to model the physical world. The OSM Web Wizard extracts a real map from OSM and creates a scenario with node itineraries.

Using these tools, first, a static SNR map is created by the Map processing module using information from the OSM map. Then, with this information, the Node processing module generates UEs from the SUMO simulation nodes and calculates their SNR traces. The Network traffic generation module then creates a traffic trace for each UE. Finally, the Dataset aggregation module aggregates the SNR and traffic traces to one aggregated trace. The following sections detail each module.

\subsection{SUMO's OSM Web Wizard}

This work uses OSM Web Wizard to generate activity for the SUMO simulations and extract the information of a real map through OSM. 
The map information, which includes information of the existing buildings, is given to the Map processing. The activity generated for the SUMO simulation is fed to SUMO through the form of a SUMO scenario, which contains the pedestrians and vehicles to be simulated along with their respective itineraries.

\subsection{SUMO}

With the scenario as input, SUMO generates mobility traces of vehicles and pedestrians which are exported in SUMO's Floating car Data (FCD) file format ${ }^{2}$ that includes information such as position, speed, and direction angle. The traces are fed to the Node processing module for the generation of UEs and calculation of fast fading models.

\subsection{Map processing}

The Map processing module generates a network topology and calculates a map with base SNR values for each point in the map, the static SNR map.

3.4.1 Network topology: cell placement. The generation of the network topology consists in placing base stations and cells over the physical map. The base stations, and respective cell direction, are located according to a report by 3rd Generation Partnership Project (3GPP) on channel models [12]. According to the document, the cells make a hexagonal grid and each base station includes 3 cells $120^{\circ}$ apart. An example is illustrated in Figure 2, where Intersite Distance (ISD) is the distance between two base stations or sites. The ISD depends on the environment being modeled. For instance, in the Urban Macro (UMa) environment, the ISD is $500 \mathrm{~m}$ [13].

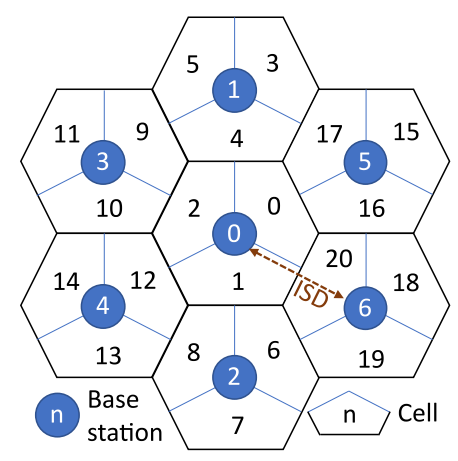

Figure 2: Cell placement.

3.4.2 Static SNR map generation. Our dataset must include the SNR traces of all nodes over time. However, to select handover areas, we create static SNR maps for each cell. A single map aggregates the information of the highest SNR from all the cells at each position. The map also includes the cell that achieves that SNR.

The process starts by creating a grid map for each cell with the SNR received at different points $10 \mathrm{~m}$ apart from each other. Points that are farther than $I S D \sqrt{3} / 2$ of any cell are not considered. For the SNR calculation we used equation 1 . For each transmitting $(t x)$

${ }^{2}$ FCD format: https://sumo.dlr.de/docs/Simulation/Output/FCDOutput.html, last access July 2021. or receiving $(r x)$ node, $P_{t x}$ represents the transmission power, $A^{\prime \prime}$ the directional antenna gain, $N F$ the noise figure.

$$
\begin{aligned}
S N R= & P_{t x}+A_{t x}^{\prime \prime}\left(\theta^{\prime \prime}, \phi^{\prime \prime}\right)+A_{r x}^{\prime \prime}\left(\theta^{\prime \prime}, \phi^{\prime \prime}\right) \\
& -P L-S F-N F_{r x}-T N-10 \log (B W)
\end{aligned}
$$

As the antennas of the UE and the cell differ, we have to perform a different calculation for the UE and the cell. The values of the transmission power $\left(P_{t x}\right)$, directional antenna gain $\left(A^{\prime \prime}\right)$, noise figure $(N F)$, thermal noise $(T N)$, and carrier bandwidth $(B W)$ depend on the considered environment and configuration ${ }^{3}$. The values for these parameters are taken from the International Telecommunication Union - Radiotelecommunication sector (ITU-R) report on simulation guidelines of radio interface technologies [13], which has much information that coincides with the 3GPP report [12].

The physical map provides relevant insights for path loss $(P L)$ and slow fading $(S F)$ calculation. Using the information extracted from the OSM map, we can determine the points of the map that are in Line of Sight (LoS) with the cell, without LoS (nLoS), or inside a building (IN). Note that line-of-sight positions do not have any building obstructing the direct line that connects that position to the cell's central position. The propagation characteristics are different in these cases, and the path loss and slow fading vary accordingly. The path loss and slow fading to apply also depend on the simulated environment and can be consulted in the 3GPP channel model report [12]. For the model of path loss inside a building, the Outdoor-to-Indoor (O2I) building penetration loss for single-frequency simulations is selected. It is of note that the slow fading, or shadow fading, is a randomly generated value. Its distribution and parameters also depend on the environment.

As an example, we used our generator in an UMa scenario using the OSM map of a part of the Berlin city map near the Reichtag. For the generation, we used the environment Urban Macro-URLLC (Configuration A) of the 3GPP and ITU-R reports with seven base stations, i.e. 21 cells. Figure 3 illustrates the global map of our piece of Berlin city map in the form of a heat map with the values of the best SNR value in $\mathrm{dBs}$ for each point. The $\mathrm{X}$ and $\mathrm{Y}$-axis are the coordinates in meters, where the position $(0,0)$ is the center of the base station 0 .

\subsection{Node processing}

The Node processing module generates the UEs based on the SUMO traces. It also generates static nodes, which do not exist in the SUMO simulation. Following that, the module calculates the SNR of each UE over time using by adding calculated fast fading values to the static SNR according to the UE position at each time.

3.5.1 UE generation. The UEs are generated based on the SUMO traces. SUMO can have different types of nodes, from which we fit into two main groups for the generation: pedestrian and vehicle. Each pedestrian is associated with a UE. Vehicles are in themselves a UE, for Vehicle-to-everything (V2X) applications, and include as many UEs as passengers inside. The number of passengers is taken from a uniform distribution. Additionally, static UEs are dispersed along with the map randomly following a uniform distribution for the values of both horizontal axes within the bounds of the map

\footnotetext{
${ }^{3}$ The UE has an isotropic antenna gain, only the cell source has a directional gain.
} 


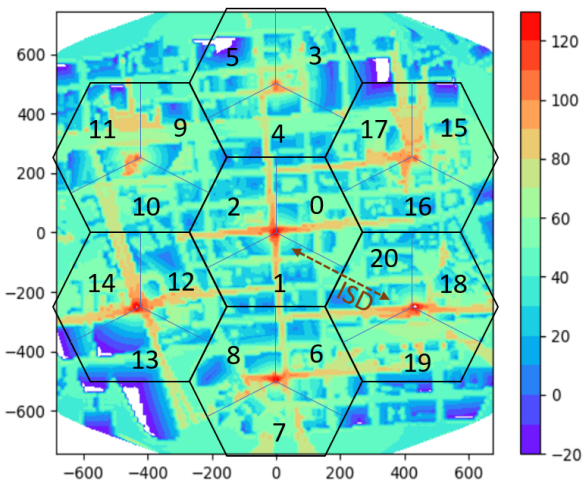

Figure 3: Generated heat map with numerated cells. The values of the SNR are in dBs.

generated by the Map generation module. The positions are regenerated so that a configured percentage of the static UEs is inside buildings and the remaining outside. In total there are four UE node types: pedestrian, vehicle, passenger, and static. If a UE leaves the considered area (farther than $I S D \sqrt{3} / 2$ of any cell) and re-enters, a new identifier is given to it. As an example, in the generated UMa scenario of Berlin, the parameters used for the UE generation are listed in Table 1.

Table 1: UE generation parameters.

\begin{tabular}{|r|c|}
\hline Parameter & Value/Distribution \\
\hline Number of static UEs & 10245 \\
\hline Static UE location & continuous uniform within bounds \\
\hline Static UEs inside (\%) & 80 \\
\hline Passengers per vehicle & discrete uniform $(1,4)$ \\
\hline
\end{tabular}

3.5.2 Fast fading model. Following the UE generation, our generator calculates the SNR of each UE over time by adding the fast fading to the SNR value of the UE position at each time. The Node processing module creates one SNR trace for each UE. The SNR values vary over time depending on the UE position and the impact of the modeled fast fading.

Let us now describe the process that we follow to model fast fading. For simplification due to the number of samples generated, multi-path fading is simulated using a simple single antenna configuration. For that, the Method of Exact Doppler Spread (MEDS) [18] is used to simulate the signal envelope under a Rician channel. One or more channels are generated from each movement trace since the model simulates static scenarios, for instance, with the same speed and obstruction level. The generator divides the movement trace into different channels depending on the variability of the speed, direction, or obstruction level of the UE. Following the nomenclature from the book where the MEDS implementation was used [19], the parameters for each generated channel are displayed in Table 2.
Table 2: MEDS parameters for each channel.

\begin{tabular}{|r|r|c|}
\hline \multicolumn{2}{|l|}{ Parameter } & Value \\
\hline \multicolumn{2}{|c|}{$N$} & 200 \\
\hline \multirow{2}{*}{$\rho$} & $T_{s}(\mathrm{~s})$ & $1 \times 10^{-3}$ \\
\cline { 2 - 4 } & $\mathrm{LoS}$ & $\sqrt{K /(K+1)}$ \\
\hline \multirow{2}{*}{$\sigma_{0}$} & $\mathrm{nLoS}$ & 0 \\
\cline { 2 - 4 } & $\mathrm{LoS}$ & $\sqrt{1 /[2(K+1)]}$ \\
\hline & $\mathrm{nLoS}$ & $\sqrt{1 / 2}$ \\
\hline & $f_{\rho}(\mathrm{Hz})$ & $f_{\max } \cos \Phi$ \\
\hline$\theta_{\rho}(\mathrm{rad})$ & $-2 \pi d_{3 D} f_{c} / c$ \\
\hline \multicolumn{2}{|c|}{$\sigma^{2}$} & $10^{K_{d B} / 10}, K_{d B} \sim \mathcal{N}\left(\mu_{k}, \sigma_{k}^{2}\right)$ \\
\hline
\end{tabular}

The states of the UE have an impact on the generation of the Rician channel. So, the UE's speed (v), the horizontal angle between the movement and direction to the cell source $(\Phi)$, the obstruction level, and the distance between the cell source and the UE $\left(d_{3 D}\right)$ must be known. The generation of a new channel is triggered when the obstruction level or the connected cell changes or when the UE's speed or direction varies by $10 \%$. The values for generating $\mathrm{K}$ $\left(\mu_{k}\right.$ and $\left.\sigma_{k}^{2}\right)$ are taken from the 3GPP report [12] and are specific to each simulation environment.

One additional element is added to model the SNR of a UE: the path loss inside a vehicle. This additional obstruction to the signal is also taken from the mentioned 3GPP report [12]. The value to be added is randomly generated per passenger and follows the normal distribution $\mathcal{N}(9,5)$.

After the SNR is calculated, the used Modulation Coding Scheme (MCS) by the UE can be calculated. The choice of MCS, according to a node's signal quality, uses the same process that is used by the NS-3 network simulator [17]. The process consists in converting the signal quality of a UE $u, S N R_{u}$, to the corresponding spectral efficiency, $\eta_{u}$, according to the Shannon theorem [20], as described in equation 2. To better adapt the result to a real environment, there is the coefficient $\Gamma=1.5$, dependent on the target Bit Error Rate (BER) $=10^{-5}$. The MCS is taken from a table from the 3GPP standard [21], where the MCS with the highest spectral efficiency lower than $\eta_{u}$ is selected. The MCS tables used are Table 6.1.4.1-1 (downlink), and Table 5.1.3.1-2 (uplink) of the 3GPP specification [21]. If the calculated $\eta_{u}$ is below the spectral efficiency of the minimum MCS value possible, the MCS has attributed a missing value, i.e. NaN.

$$
\begin{gathered}
\eta_{u}=\log _{2}\left(1+\frac{S N R_{u}}{\Gamma}\right) \\
\Gamma=-\frac{\ln (5 \cdot B E R)}{1.5}
\end{gathered}
$$

3.5.3 Output. The SNR traces contain measures of the SNR value over sample periods of $1 \mathrm{~ms}$. They are composed by the columns:

- Time: the timestamp of the SNR measure;

- UE: the UE with the SNR measure;

- X: X position according to the SUMO map;

- Y: Y position according to the SUMO map;

- SNR: calculated SNR at instant Time;

- MCS: the MCS used according to the SNR;

- Cell: the cell which the UE is attached to. 
Table 3: Traffic generation parameters.

\begin{tabular}{|c|c|c|c|c|c|c|}
\hline Type & Component & Distribution & Parameters / Values & Direction & Slice & Comment \\
\hline \multirow{2}{*}{ MTC } & Inter packet arrival (s) & Exponential & $1 / \lambda=234$ & \multirow{2}{*}{ Uplink } & \multirow{2}{*}{ mMTC } & \multirow{2}{*}{$\begin{array}{l}\text { Negative size: new } \\
\text { random generation. }\end{array}$} \\
\hline & $i^{\text {th }}$ packet size (B) & Normal & $\mu=200 ; \sigma=60$ & & & \\
\hline \multirow{2}{*}{ V2I (CAM) } & Inter packet arrival (s) & Truncated normal & $\begin{array}{c}\mu=0.4 ; \sigma=1 ; \min =0.1 \\
\max =1\end{array}$ & \multirow{2}{*}{ Uplink } & \multirow{2}{*}{ URLLC } & \multirow{2}{*}{$\begin{array}{c}\text { Base in CAM message } \\
\text { report [22] }\end{array}$} \\
\hline & $i^{\text {th }}$ packet size (B) & Truncated normal & $\begin{aligned} \mu & =400 ; \sigma=200 ; \\
\min & =200 ; \max =500\end{aligned}$ & & & \\
\hline \multirow{4}{*}{$\begin{array}{r}\text { V2I } \\
(\mathrm{DENM})\end{array}$} & $\begin{array}{r}\text { Inter burst occurrence } \\
\text { time (s) }\end{array}$ & Exponential & $1 / \lambda=60$ & \multirow{4}{*}{ Uplink } & \multirow{4}{*}{ URLLC } & \multirow{4}{*}{$\begin{array}{l}\text { Geo-located burst } \\
\text { occurrences trigger } \\
\text { messages. }\end{array}$} \\
\hline & Burst position $(\mathrm{m})$ & Uniform & Within map bounds & & & \\
\hline & Burst radius $(\mathrm{m})$ & Fixed & 50 & & & \\
\hline & Packet size (B) & Fixed & 320 & & & \\
\hline \multirow{5}{*}{$\begin{array}{l}\text { Offline } \\
\text { Video }\end{array}$} & Inter packet arrival (s) & Log-normal & Fitted to type & \multirow{5}{*}{ Downlink } & \multirow{5}{*}{ eMBB } & \multirow{5}{*}{$\begin{array}{l}\text { Types correspond to real } \\
\text { traces. Possible types: } \\
\text { music video, sports } \\
\text { compilation, writing, } \\
\text { cooking, reaction. }\end{array}$} \\
\hline & Clip duration (s) & Normal & $\begin{array}{l}\mu=d u r(\text { type }) \\
\sigma=0.3 * d u r(\text { type })\end{array}$ & & & \\
\hline & $i^{\text {th }}$ Packet size (B) & Normal & $\begin{array}{c}\mu=\operatorname{size}(\text { type }, i) ;^{4} \\
\sigma=0.3 * \operatorname{size}(\text { type }, i)\end{array}$ & & & \\
\hline & Inter session time (s) & Exponential & $1 / \lambda=3600$ & & & \\
\hline & Session duration (s) & Exponential & $1 / \lambda=1200$ & & & \\
\hline \multirow{7}{*}{ Web } & Inter session time (s) & Exponential & $1 / \lambda=600$ & \multirow{7}{*}{ Downlink } & \multirow{7}{*}{ eMBB } & \multirow{7}{*}{$\begin{array}{c}\text { Each web session } \\
\text { consists in a given } \\
\text { number of pages. Each } \\
\text { page contains a given } \\
\text { number of objects [23]. }\end{array}$} \\
\hline & Pages per session & Normal & $\mu=3.86 ; \sigma=2.465$ & & & \\
\hline & Page size (B) & Truncated log-normal & $\begin{array}{c}\mu=8.37 ; \sigma=1.37 \\
\min =100 ; \max =20 * 10^{6}\end{array}$ & & & \\
\hline & Number of objects & Truncated Pareto & $\begin{array}{l}\alpha=1.11 ; k=2 ; \\
\min =0 ; \max =53\end{array}$ & & & \\
\hline & Object size (B) & Truncated log-normal & $\begin{array}{c}\mu=2.36 ; \sigma=6.17 \\
\min =50 ; \max =20 * 10^{6}\end{array}$ & & & \\
\hline & Parsing time (s) & Exponential & $1 / \lambda=0.13$ & & & \\
\hline & Reading time (s) & Exponential & $1 / \lambda=3$ & & & \\
\hline \multirow[b]{3}{*}{ VoIP } & Inter call arrival (s) & Exponential & $1 / \lambda=600$ & \multirow[b]{3}{*}{ Both } & \multirow[b]{3}{*}{ eMBB } & \multirow{3}{*}{$\begin{array}{l}\text { When active a } 40 \text { byte } \\
\text { packet is sent every } \\
20 \mathrm{~ms} \text {, otherwise a } 16 \\
\text { byte packet is sent every } \\
160 \mathrm{~ms} \text { [23]. }\end{array}$} \\
\hline & Call duration (s) & Exponential & $1 / \lambda=180$ & & & \\
\hline & Voice activity & $\begin{array}{l}\text { Markov Chain w/ } \\
\quad \text { sampling } T\end{array}$ & $\begin{array}{c}p(\text { on } \mid \text { off })=0.01 \\
p(\text { off } \mid \text { on })=0.01 \\
T=0.02\end{array}$ & & & \\
\hline
\end{tabular}

\subsection{Network traffic generation}

Before completing the dataset generation, the last task is generating data traffic. The generated traffic is modeled after common network applications such as web browsing and video playback. It also simulates vehicular applications and machine communications that are among the new applications for mobile wireless networks with the introduction of 5G [10]. To boost its applicability in 5G scenarios, we also attribute network slice identifiers to each traffic type. The generated traffic is summarized in Table 3.

3.6.1 Traffic generation. The network traffic for the synthetic dataset is generated based on a set of traffic classes that aim to emulate different applications expected to be part of a $5 \mathrm{G}$ mobile wireless network. These classes are Machine Type Communications (MTC) sensors, offline video, vehicle-based information, VoIP, and Web.

${ }^{4}$ For index $i$ values higher than the type's number of packets the index circulates over the last $2 / 3$ of the packets.
MTC sensors. MTC sensors are emulated using an exponentially distributed inter-packet time and a truncated normal distribution for the packet size. In a survey on traffic models by Navarro-Ortiz et al.[24], this was identified as a recurrent traffic model appointed by different entities such as the 3GPP.

Offline video. Offline video is simulated using a different approach than a simple Constant Bitrate (CBR) transmission. Through observation of traces from Youtube sessions, we noticed chunks of the video are loaded depending on the playback offset position, this translates more to a more burst behavior than CBR. To simulate offline video, we used network information from the traces generated by watching Youtube videos with uninterrupted playback. A set of videos ranging from sports to music or simply popular reaction videos was collected and set as possible templates. From these templates, the inter-packet arrival is fitted to a log-normal distribution using Python's library scipy. We define the video duration using a 
normal distribution based on the template clip duration and each packet length from a normal distribution based on the template's packet length. We repeated the last $2 / 3$ of the packet length list to accommodate generated video traces with a higher duration than the template duration. We observed that the first packets had considerably higher lengths to provide a faster initial load of the video clip, and so we use only the last values for this repetition. Table 4 lists the used templates, and their Maximum Likelihood Estimates (MLEs) of the parameters in the log-normal distribution fit if the Inter Packet Arrival (IPA) and clip duration. Additionally, Figure 4 illustrates the size distribution of the youtube chunks. As can be observed, a considerable amount of 1.5 to 2 Mbyte chunks are sent, as well as some lower-sized chunks (around 500KBytes), which is also a consequence of the video codification.

Table 4: IPA distribution parameters and duration for each template

\begin{tabular}{|r|c|c|c|c|}
\hline \multirow{2}{*}{ Template } & \multicolumn{3}{|c|}{ IPA - parameter's MLE } & $\begin{array}{c}\text { Duration } \\
\text { (minutes) }\end{array}$ \\
\cline { 2 - 4 } & Shape & Location & Scale & $21: 21.701$ \\
\hline News & $4.84 \times 10^{-3}$ & $3.28 \times 10^{2}$ & $3.31 \times 10^{2}$ & $07: 37.381$ \\
\hline Cooking & 1.61 & $3.48 \times 10^{-3}$ & 1.79 & $04: 00.501$ \\
\hline Music & 2.40 & $2.84 \times 10^{-3}$ & $8.72 \times 10^{-1}$ & $16: 38.661$ \\
\hline Writing & $3.41 \times 10^{-1}$ & -4.45 & 8.15 & $12: 38.401$ \\
\hline Reaction & $1.51 \times 10^{-1}$ & $-1.92 \times 10^{1}$ & $2.37 \times 10^{1}$ & $10: 07.041$ \\
\hline Sports & $4.70 \times 10^{-1}$ & -4.21 & 8.75 & \\
\hline
\end{tabular}

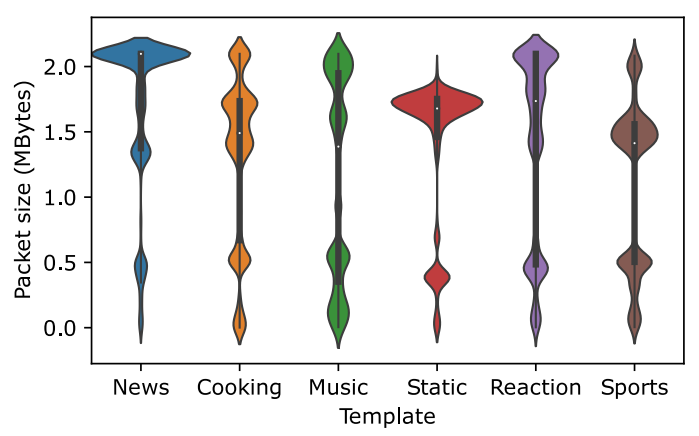

Figure 4: Distribution of youtube chunk sizes by the template.

Vehicle-based information. Vehicle-based communication portraits Vehicle-to-Infrastructure (V2I) communications. The traffic generation uses periodical and emergency traffic. To generate periodical traffic, we use a random distribution, which configuration is based on a report of Cooperative Awareness Message (CAM) messages by Car 2 Car Communication Consortium (C2C-CC) [22]. For emergency traffic, we generate a random region-of-interest that forces nodes within that area to trigger a Decentralized Environmental Notification Message (DENM) packet. This region-of-interest has a circular shape, and we used a uniform distribution for a center with a fixed radius. The time in between the occurrence of bursts is exponentially distributed.
VoIP. The traffic model for VoIP is a model with silence suppression according to 3GPP guidelines [23]. The model follows a conversation on-off switch to dictate when a person is talking (and traffic is flowing normally) or not. In addition to the 3GPP model, the inter-call and duration times are sampled from exponential distributions.

Web. Web traffic also follows a traffic model from 3GPP guidelines [23], where a user requests web pages that are processed and require their specific objects. After a given reading time from the last web page request, a new web page is requested.

3.6.2 Usage scenarios. For the usage scenario, our generator defines the type of traffic sources that each type of UE can generate, but also the slices that are created and the traffic scenarios.

Traffic sources. Traffic is distributed to the different node types according to a defined traffic source type depending on who or what is operating the UE: a person using general broadband services, a vehicle sending CAM and DENM messages, and a machine sending sensor information uplink. The mapping between source types to node types and the equivalent traffic types used is listed in Table 5.

Table 5: Traffic type allocated to each node type

\begin{tabular}{|r|c|c|}
\hline Source type & Node type & Traffic \\
\hline Person & $\begin{array}{c}\text { Passenger, } \\
\text { Pedestrian, } \\
60 \% \text { Static }\end{array}$ & $\begin{array}{c}20 \% \text { Idle, 20\% Offline Video, } \\
20 \% \text { VoIP, 40\% Web }\end{array}$ \\
\hline Vehicle & Vehicle & V2I (CAM+DENM) \\
\hline Machine & $40 \%$ Static & MTC \\
\hline
\end{tabular}

Traffic slices. The traffic of the different applications are grouped in different slices. We decided to create three slices: one slice for enhanced Mobile BroadBand (eMBB), one for Ultra Reliable Low Latency Communication (URLLC), and another one for massive Machine Type Communication (mMTC). The organization is illustrated in Table 3.

3.6.3 Output. The traffic traces are composed traces recorded at the timestamps when a new traffic packet is triggered. They are composed by:

- Time: Timestamp of the triggered traffic packet;

- UE: the traffic source or destination;

- Direction: uplink or downlink;

- Bytes: length of the packet in bytes;

- Types: The types of traffic used by the UE;

- Slice: slice of the traffic.

\subsection{Dataset aggregation}

Since the SNR traces have a fine time period of $1 \mathrm{~ms}$, and to have a workable dataset for some applications, a new aggregated dataset is generated containing aggregated information of both the SNR and traffic traces within time windows of a configurable time, we set the default to $10 \mathrm{~s}$. 
3.7.1 Scheduling simulation. With big packet sizes or small-time windows, there is the probability that the queued traffic is not sent in its entirety within the aggregation time window. So, we had to account for the unscheduled traffic and add it to the traffic of the next time window. For this, a scheduling process is applied to simulate a blind equal throughput scheduler [25] per slice. To provide the scheduling, we need to stipulate a resource limitation per time window. To that effect, targets for the required throughput of each slice were specified, and at each time window, the number of resources required to reach that target was used for the resource limitation. The default target values are listed in Table 6.

Table 6: Targets considered for scheduling

\begin{tabular}{|r|r|c|}
\hline Slice & Direction & Target (Mbps) \\
\hline eMBB & DL & 500 \\
\hline URLLC & UL & 10 \\
\hline eMBB & UL & 2 \\
\hline mMTC & UL & 0.01 \\
\hline
\end{tabular}

3.7.2 Filtering entries. The entries where the UEs have a missing value, $N a N$, for MCS are removed at this point since the UE is considered to have no radio connection. Also, at this point, there is the possibility to filter some nodes to balance the static vs mobile UE relation.

3.7.3 Output. The aggregated trace is composed of the columns:

- Time: time of the beginning of the time window;

- UE: the UE the dataset entry is relative to;

- Cell: The cell the UE is attached to. If one UE has been in two cells over one time window, there is a UE entry for each cell;

- Slice: the slice of the UE the dataset entry is relative to;

- Bytes: the sum of packet lengths generated within the time window;

- Bytes Queued: the sum of bytes in the UE queue before scheduling within the time window;

- Bytes Scheduled: the sum of bytes scheduled within the time window;

- SNR: the average SNR within the time window;

- MCS: MCS calculated with the average SNR as input;

- SE: spectral efficiency of the calculated MCS;

- RBs: the number of Resource Blocks (RBs) required to schedule the summed packet lengths;

\section{GENERATED DATASET VISUALIZATION}

As referred above, we used the dataset generator to generate an UMa scenario using the OSM map of a part of the Berlin city map near the Reichtag. This section describes its generation, provides some visualization in the generated data, and explores customization possibilities.

\subsection{Dataset parameterization}

The parameters used for the generation other than the mentioned default configurations are listed in Table 7 . The SUMO version used is 1.7.0. The SUMO simulation and dataset duration were configured to 23200 seconds, over 6 hours. A higher duration would require a more detailed trip activity generation to create scenarios with periodic commute travels and with separate weekdays from weekend activity. A lower duration would decrease the variety of available information. For the activity generation using the OSM Web Wizard, the values of Through Traffic Factor (TTF) and count are specified for each vehicle type used are listed in the table. TTF controls how the traffic generation is more or less geared towards the center, while count controls the number of nodes generated per time and space. In the scope of the introduced generator, the bicycles were considered pedestrians. The environment selected for SNR calculation was the Urban Macro-URLLC with Configuration A. Upon the dataset aggregation, the number of static UES was 5000. After the initial generation of UEs, the ratio of static to mobile UEs was 10:1. We decided to reduce it to around 5:1, but it ultimately depends on the scenario we are trying to simulate.

Table 7: Dataset generation parameters

\begin{tabular}{|c|c|c|c|}
\hline Module & \multicolumn{2}{|c|}{ Parameter } & Value \\
\hline SUMO & \multicolumn{2}{|c|}{ Duration (s) } & 23200 \\
\hline \multirow{6}{*}{$\begin{array}{r}\text { OSM Web } \\
\text { Wizard }\end{array}$} & \multirow{2}{*}{ Car } & TTF & 5 \\
\hline & & Count & 12 \\
\hline & \multirow{2}{*}{ Pedestrian } & TTF & 1 \\
\hline & & Count & 10 \\
\hline & \multirow{2}{*}{ Bicycle } & TTF & 1 \\
\hline & & Count & 10 \\
\hline \multirow{2}{*}{$\begin{array}{r}\text { Map and Node } \\
\text { processing }\end{array}$} & \multicolumn{2}{|c|}{ Environment } & $\begin{array}{c}\text { UMa-URLLC, } \\
\text { Conf. A }\end{array}$ \\
\hline & \multicolumn{2}{|c|}{ Base stations } & 21 \\
\hline $\begin{array}{r}\text { Dataset } \\
\text { aggregation }\end{array}$ & \multicolumn{2}{|c|}{ UE filter } & $\begin{array}{l}\text { Max. } 5000 \\
\text { static UEs }\end{array}$ \\
\hline
\end{tabular}

\subsection{Dataset characterization}

This section provides a characterization of the dataset regarding the generated UEs and activity in each cell.

4.2.1 UE characterization. The total number of generated UEs is listed in Table 8. This high UE count gives a high number of inputs required by machine learning algorithms. However, the static UE count does not match the 5000 set maximum because some of the 5000 filtered UEs had a low SNR. It happens mainly in the buildings in the network edges since there are no cells on the opposite side to the edge cells.

Table 8: Number of generated UEs

\begin{tabular}{|r|c|}
\hline Car passengers & 16609 \\
\hline Cars & 8507 \\
\hline Static & 4540 \\
\hline Pedestrian & 13956 \\
\hline Total & $\mathbf{4 3 6 1 2}$ \\
\hline
\end{tabular}

Static UEs have the duration of the entire generation time. Mobile UEs have the duration of their relative SUMO nodes within the 
bounds of the dataset generator, i.e. within ISD of the nearest cell. The distribution of the duration of every type of UE is represented in Figure 5. Pedestrian UEs have the highest duration since they take more time during their trips, with a median value of around $500 \mathrm{~s}$. Car passengers have the same distribution as the respective vehicle since they are generated and removed simultaneously.

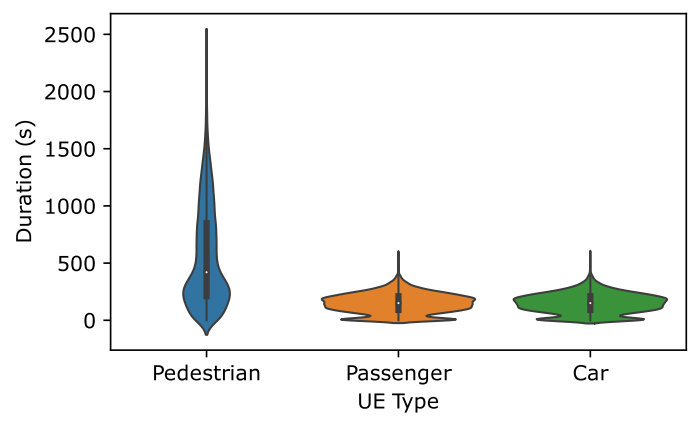

Figure 5: Trace duration distribution by UE type.

4.2.2 Cell characterization. Regarding the generated aggregate dataset, the amount of data contained in each cell can be looked up in Figure 6, which presents a representation of the number of connected UEs and sent/received traffic at each time window. For each cell and direction, at each time window, the number of UEs present is counted, and the number of bytes sent/received is summed. Each dot represents the averages of the values of each time window for each cell and direction. As can be observed regarding uplink, the cells with more information are cells 0 , 9, and 12, with a high count of UEs and traffic. Regarding the uplink, the same cells are on top regarding UE and traffic count, but the distribution is different. For instance, in the uplink cells, 9 and 12 send/receive sensibly the same amount of traffic.

To observe the SNR distribution at each cell, Figure 7 presents the distribution of the SNR average values for each time window. As can be observed, the different cells have different distributions due to the different obstacles and movements within each cell. With this distinct distribution, machine learning algorithms that aim to adapt to different cells can benefit from this dataset for its validation.

\subsection{Dataset customization}

A significant drawback of using synthetic datasets to train machine learning is the lack of accuracy in modeling the physical world. Providing different configurations allows for a better validation of machine learning models that use synthetic data. One of the main benefits of our dataset generator is that it can be customized to explore variations of the default parameters of the dataset. This section introduces customizations on the SNR and generated traffic.

4.3.1 Radio signal quality. One possible way of customizing the dataset is changing the radio signal quality behavior by modifying the attenuation values used. Figure 8 illustrates the SNR distribution of the aggregate trace when adding an increasing attenuation of 10 and $30 \mathrm{~dB}$. The attenuation is added in the Map processing module, and a new aggregate dataset is generated for each attenuation

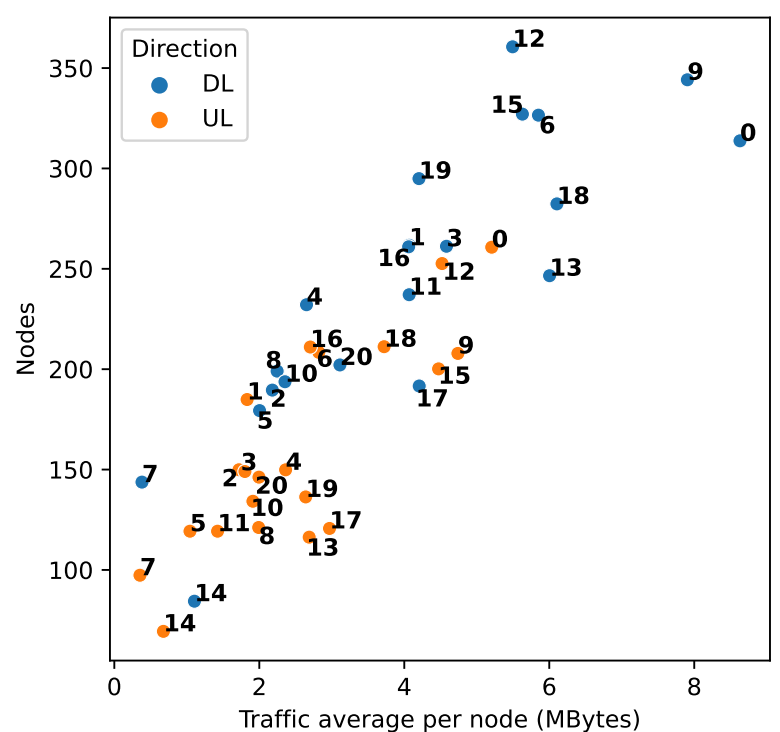

Figure 6: Average UE count and traffic sent by each UE.

configuration. The configured attenuation values result in different distributions of the SNR.

4.3.2 Traffic scenarios. To consider different traffic scenarios, different traffic behaviors were created in the traffic of the eMBB slice. Besides the default generation in Table 3, different parameterization is specified to originate an occasional and constant behavior. The parameters used for each behavior are listed in Table 9.

Table 9: Parameter alterations for different traffic behaviors.

\begin{tabular}{|c|c|c|c|}
\hline Traffic & Behavior & Component & Value \\
\hline \multirow{6}{*}{$\begin{array}{l}\text { Offline } \\
\text { Video }\end{array}$} & \multirow{2}{*}{ Default } & Inter session time $(\mathrm{t})$ & $1 / \lambda=1200$ \\
\hline & & Session duration $(\mathrm{t})$ & $1 / \lambda=1200$ \\
\hline & \multirow{2}{*}{ Occasional } & Inter session time $(\mathrm{t})$ & $1 / \lambda=2400$ \\
\hline & & Session duration $(\mathrm{t})$ & $1 / \lambda=600$ \\
\hline & \multirow{2}{*}{ Constant } & Inter session time $(\mathrm{t})$ & $1 / \lambda=0$ \\
\hline & & Session duration $(\mathrm{t})$ & $1 / \lambda=1200$ \\
\hline \multirow{6}{*}{ VoIP } & \multirow{2}{*}{ Default } & Inter call arrival (t) & $1 / \lambda=600$ \\
\hline & & Call duration $(\mathrm{t})$ & $1 / \lambda=180$ \\
\hline & \multirow{2}{*}{ Occasional } & Inter call arrival (t) & $1 / \lambda=1200$ \\
\hline & & Call duration $(\mathrm{t})$ & $1 / \lambda=60$ \\
\hline & \multirow{2}{*}{ Constant } & Inter call arrival (t) & $1 / \lambda=10$ \\
\hline & & Call duration $(\mathrm{t})$ & $1 / \lambda=180$ \\
\hline \multirow{3}{*}{ Web } & Default & Inter session time $(\mathrm{t})$ & $1 / \lambda=600$ \\
\hline & Occasional & Inter session time $(\mathrm{t})$ & $1 / \lambda=1200$ \\
\hline & Constant & Inter session time $(\mathrm{t})$ & $1 / \lambda=10$ \\
\hline
\end{tabular}

With these traffic behavior, we created three scenarios: The standard, which exclusively uses the default behavior; the sporadic, which exclusively uses the occasional behavior, and the undistributed that uses a combination of behaviors to provide a scenario where a smaller number of UEs is responsible for more traffic. The scenarios are specified in Table 10. 


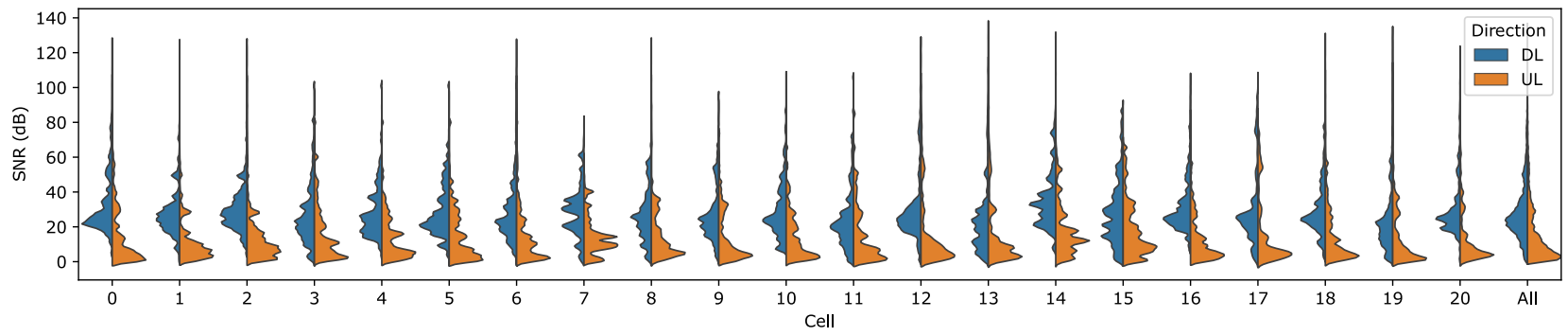

Figure 7: SNR distribution per cell.

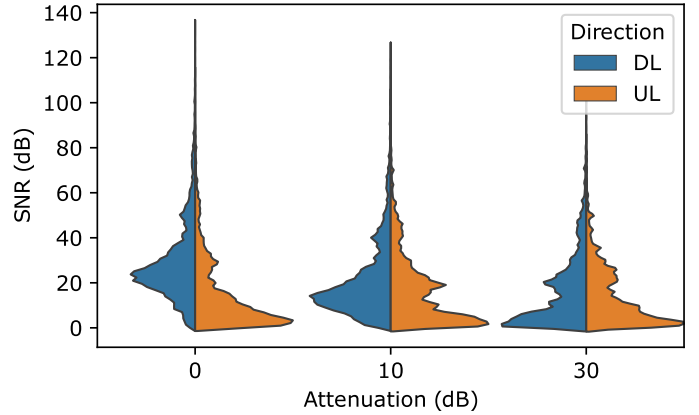

Figure 8: SNR distribution using different levels of attenuation.

Table 10: Traffic behavior used in each scenario

\begin{tabular}{|r|c|}
\hline Scenario & Traffic behaviors \\
\hline Standard & $100 \%$ Default \\
\hline Sporadic & $100 \%$ Occasional \\
\hline Undistributed & $25 \%$ Idle, $25 \%$ Occasional, $50 \%$ Constant \\
\hline
\end{tabular}

\section{CONCLUSION}

This work introduces a synthetic dataset generation tool for wireless mobile networks. The generation is made using an urban mobility simulator, SUMO [11], and empirical channel models for the SNR calculation [12][13]. For the traffic modeling, we used existent models for traffic generation [23], but also created models based on reports containing statistics of V2X messages [22], and based on collected YouTube traffic.

This dataset generator aims to solve the problem of the lack of extensive datasets in the context of UE activity and radio signal connection in wireless mobile networks. The introduced generator can be used by machine learning solutions to solve problems of resource allocation in the edge, where a complete knowledge of the UE activity and movement is required. The dataset generation procedure can be done iteratively following the decisions made by a reinforcement learning algorithm, as opposed to the fixed datasets with real traces.

One dataset was generated consisting of more than $6 \mathrm{~h}$ with over 40000 UEs spread over 21 cells, where there are, on average, simultaneously hundreds of UEs per cell. The static UEs have the entirety of the dataset duration, and the mobile UEs have traces up to $2500 \mathrm{~s}$.

Future work in dataset generation can integrate the dataset generation with more SUMO features such as activity generation where habitation and working zones are defined. With such activity, datasets could be created with information from entire weeks to explore the periodicity of commute trips. Another welcome extension is the generation of the dataset with real base station locations and cell directions, and the consideration of terrain elevation. After these, comparison with real datasets can be done and adjustments to the synthetic generated dataset can be applied.

\section{ACKNOWLEDGMENTS}

This work was supported by national funds through Fundação para a Ciência e a Tecnologia (FCT) with reference UIDB/50021/2020 and SFRH/BD/132053/2017.

\section{REFERENCES}

[1] Ljupco Jorguseski, Adrian Pais, Fredrik Gunnarsson, Angelo Centonza, and Colin Willcock. Self-organizing networks in 3gpp: standardization and future trends. IEEE Communications Magazine, 52(12):28-34, 2014.

[2] Bego Blanco, Jose Oscar Fajardo, Ioannis Giannoulakis, Emmanouil Kafetzakis, Shuping Peng, Jordi Pérez-Romero, Irena Trajkovska, Pouria S. Khodashenas, Leonardo Goratti, Michele Paolino, Evangelos Sfakianakis, Fidel Liberal, and George Xilouris. Technology pillars in the architecture of future $5 \mathrm{~g}$ mobile networks: Nfv, mec and sdn. Computer Standards \& Interfaces, 54:216-228, 2017. SI: Standardization SDN\&NFV.

[3] Yongjun Xu, Guan Gui, Haris Gacanin, and Fumiyuki Adachi. A survey on resource allocation for $5 \mathrm{~g}$ heterogeneous networks: Current research, future trends, and challenges. IEEE Communications Surveys Tutorials, 23(2):668-695, 2021.

[4] Vaclav Raida, Philipp Svoboda, Martin Koglbauer, and Markus Rupp. On the stability of rsrp and variability of other kpis in lte downlink - an open dataset. In GLOBECOM 2020 - 2020 IEEE Global Communications Conference, pages 1-6, 2020.

[5] Ayub Bokani, Mahbub Hassan, Salil S. Kanhere, Jun Yao, and Garson Zhong. Comprehensive mobile bandwidth traces from vehicular networks. In Proceedings of the 7th International Conference on Multimedia Systems, MMSys '16, New York, NY, USA, 2016. Association for Computing Machinery.

[6] Vaclav Raida, Philipp Svoboda, and Markus Rupp. Real world performance of lte downlink in a static dense urban scenario - an open dataset. In GLOBECOM 2020 - 2020 IEEE Global Communications Conference, pages 1-6, 2020.

[7] Britta Meixner, Jan Willem Kleinrouweler, and Pablo Cesar. $4 \mathrm{~g} / \mathrm{lte}$ channel quality reference signal trace data set. In Proceedings of the 9th ACM Multimedia Systems Conference, MMSys '18, page 387-392, New York, NY, USA, 2018. Association for Computing Machinery.

[8] Darijo Raca, Jason J. Quinlan, Ahmed H. Zahran, and Cormac J. Sreenan. Beyond throughput: A 4g lte dataset with channel and context metrics. In Proceedings of the 9th ACM Multimedia Systems Conference, MMSys '18, page 460-465, New York, NY, USA, 2018. Association for Computing Machinery.

[9] Ashish Dandekar, Remmy A. M. Zen, and Stéphane Bressan. A comparative study of synthetic dataset generation techniques. In Sven Hartmann, Hui Ma, Abdelkader Hameurlain, Günther Pernul, and Roland R. Wagner, editors, Database and 
Expert Systems Applications, pages 387-395, Cham, 2018. Springer International Publishing.

[10] NGMN. 5G White Paper. Technical report, Next Generation Mobile Networks (NGMN) Alliance, February 2015

[11] Pablo Alvarez Lopez, Michael Behrisch, Laura Bieker-Walz, Jakob Erdmann, YunPang Flötteröd, Robert Hilbrich, Leonhard Lücken, Johannes Rummel, Peter Wagner, and Evamarie Wießner. Microscopic traffic simulation using sumo. In The 21st IEEE International Conference on Intelligent Transportation Systems. IEEE, 2018.

[12] 3GPP. Study on channel model for frequencies from 0.5 to $100 \mathrm{GHz}$. Technical Report (TR) 38.901, 3rd Generation Partnership Project (3GPP), 10 2019. Version 16.0.0.

[13] ITU-R. Guidelines for evaluation of radio interface technologies for imt-2020 Report itu-r m.2412-0, ITU Radiocommunication Sector, 102017.

[14] Afonso Oliveira and Teresa Vazão. Synthetic dataset for mobile wireless networks with SUMO - individual traces, November 2021. IEEE DataPort. https://doi.org/ $10.21227 / 4 z d n-q e 46$.

[15] Afonso Oliveira and Teresa Vazão. Synthetic dataset for mobile wireless networks with SUMO - aggregated traces, November 2021. Zenodo. https://doi.org/10. 5281/zenodo.5513705.

[16] Afonso Oliveira and Teresa Vazão. Dataset generator for wireless mobile networks, November 2021. Zenodo. https://doi.org/10.5281/zenodo.5514012.
[17] Giuseppe Piro, Nicola Baldo, and Marco Miozzo. An lte module for the ns3 network simulator. Proceedings of the 4th International ICST Conference on Simulation Tools and Techniques: March 2011, 012011.

[18] M. Patzold, U. Killat, F. Laue, and Yingchun Li. On the statistical properties of deterministic simulation models for mobile fading channels. IEEE Transactions on Vehicular Technology, 47(1):254-269, 1998.

[19] M. Viswanathan and V. Mathuranathan. Wireless Communication Systems in Matlab: (Black \& White Edition). Independently Published, 2018.

[20] Proakis. Digital Communications 5th Edition. McGraw Hill, 2007.

[21] 3GPP. 5G; NR; Physical layer procedures for data. Technical Specification (TS) 38.214, 3rd Generation Partnership Project (3GPP), July 2019. Version 15.6.0.

[22] Vincent Martinez and Friedbert Berens. Survey on CAM statistics. Technical Report TR2052, Car 2 Car Communication Consortium (C2c-CC), 12 2018. Version 1.0.1.

[23] TSG-RAN1. LTE physical layer framework for performance verification. Meeting R1-070674, 3rd Generation Partnership Project (3GPP), 02 2007. 3GPP TSGRAN1\#48.

[24] Jorge Navarro-Ortiz, Pablo Romero-Diaz, Sandra Sendra, Pablo Ameigeiras, Juan J. Ramos-Munoz, and Juan M. Lopez-Soler. A survey on $5 \mathrm{~g}$ usage scenarios and traffic models. IEEE Communications Surveys Tutorials, 22(2):905-929, 2020.

[25] F. Capozzi, G. Piro, L. A. Grieco, G. Boggia, and P. Camarda. Downlink packet scheduling in lte cellular networks: Key design issues and a survey. IEEE Communications Surveys Tutorials, 15(2):678-700, Second 2013. 